\title{
Qualidade dos registros no relatório de anestesia utilizado no bloco cirúrgico de um hospital terciário de Porto Alegre- RS
}

\section{Quality of records in the anesthesia report used in the operating room of a tertiary hospital in Porto Alegre-RS}

\author{
Maristênia Machado Araújo ${ }^{1}$ \\ Maristela Machado Araújo ${ }^{2}$ \\ Fleming Salvador Pedroso ${ }^{3}$ \\ Marcello Mascarenhas ${ }^{3}$
}

\section{RESUMO}

Introdução: A complexidade dos procedimentos clínicos cirúrgicos e do diagnóstico, realizados no bloco cirúrgico, com o uso de diversos fármacos, torna-se imprescindível a existência de um registro adequado. Objetivo: Foi avaliar a qualidade dos registros em relatórios de anestesia utilizados no bloco cirúrgico de um hospital terciário. Metodologia: Para isso foi analisado o preenchimento dos relatórios de anestesia (RA), documento que acompanha o paciente desde seu acesso ao bloco cirúrgico até a completa recuperação. A pesquisa é caracterizada como quantitativa e exploratória, onde foram analisados 437 documentos. Resultado: A maioria das cirurgias (57\%) foi de média duração, predominando os pacientes do sexo feminino na faixa etária de 60 anos ou mais. Foram constatadas falhas no preenchimento de mais de $90 \%$ dos RA. As informações mais abordadas são as que registram o transcorrer da cirurgia como a técnica anestésica e tipo de cirurgia em $99 \%$ dos documentos, porém evidenciou-se reduzida avaliação pré-anestésica, fato que pode comprometer a qualidade da assistência ao paciente. Entretanto, observa-se que outras questões foram levantadas, como o uso de documentos preexistentes, sem reformulação e orientações para profissionais. Conclusão: Assim o estudo sugere novas pesquisas, como a análise do RA pelos profissionais que o utilizam, no sentido de identificar informações excessivas, inadequadas ou desnecessárias, e, consequentemente, a adequação e efetividade desse instrumento de comunicação.

\section{PALAVRAS-CHAVE}

Avaliação pré-anestésica; Pós-operatório; Qualidade de documentos hospitalares.

\footnotetext{
${ }^{1}$ Mestra em Reabilitação e Inclusão pelo Centro Universitário Metodista - IPA.

${ }^{2}$ Docente da Universidade Federal de Santa Maria.

${ }^{3}$ Docente do Centro Universitário Metodista - IPA.
} 


\section{ABSTRACT}

Introduction: The complexity of the surgical and diagnostic clinical procedures, performed in the operating room, with the use of various drugs, makes it necessary to have an adequate record. Objective: To evaluate the quality of the records in anesthesia reports used in the operating room of a tertiary hospital. Methodology: For this, anesthesia reports (AR) were analyzed or filled out, a document that accompanies the patient from their access to the operating room to complete recovery. The research is characterized as quantitative and exploratory, where 437 documents were driven. Result: Most surgeries (57\%) were of medium duration, with female patients predominating in the age group of 60 years or more. Failures were found not to fill more than $90 \%$ of the AR. The most addressed information is that which records or proceeds from surgery as the anesthetic technique and type of surgery in $99 \%$ of the documents, however there was a reduced pre-anesthetic evaluation, a fact that can compromise the quality of patient care. However, it is observed that other issues were raised, such as the use of pre-existing documents, without reformulation and guidance for professionals. Conclusion: Thus, the study required new research, such as an analysis of AR by the professionals who use it, in order to identify excessive, inadequate or unnecessary information, and, consequently, the adequacy and effectiveness of this communication instrument.

\section{KEYWORDS}

Pre-anesthetic evaluation. Postoperative. Quality documents nosocomial 


\section{INTRODUÇÃo}

O Centro Cirúrgico é uma unidade fechada e comple$\mathrm{xa}$, com risco, muitas normas e rotinas, tendo em vista o número de procedimentos técnicos à anestesia e cirurgia, propriamente. Nesse ambiente o papel do enfermeiro responsável é fundamental, o qual deve ter conhecimento científico, habilidade e entendimento sobre relações humanas, para administrar conflitos entre os diversos profissionais atuam durante a cirurgia (STUMM MAÇALAI; KIRCHNER, 2006)

A intervenção cirúrgica representa um risco à vida de qualquer pessoa, pois além da necessidade de fármacos, envolve uma carga emocional específica e diferenciada entre indivíduos. A necessidade de submeter-se a uma cirurgia gera no paciente um estresse que pode manifestar-se de várias formas, afetando a fisiologia (SOUZA; CARVALHO; PALDINO, 2011). Nesse contexto, independente do porte do procedimento cirúrgico e do tipo de anestesia, o paciente encontra-se sob risco até sua plena recuperação (SOBECC, 2013).

A avaliação pré-anestésica é um processo de avaliação clínica que antecede cuidados anestésicos, tendo como vantagem a redução da morbidade e aumento da qualidade dos procedimentos cirúrgicos (ISSA et al. 2011). Assim, as informações e resultados dos exames servem para a construção do RA, subsidiando informações relacionadas ao paciente, para outros componentes da equipe cirúrgica.

Entretanto, em algumas instituições de saúde o contato com o anestesiologista ocorre somente após o acesso do paciente no bloco cirúrgico, ocasião em que este profissional procede a avaliação pré-anestésica. Paralelamente, são utilizadas algumas escalas de avaliação de risco cirúrgico como aquela preconizada pela American Society of Anesthesiologists (ASA), que utiliza escores a fim de indicar o estado físico do paciente. A partir disso, as informações obtidas e referidas pelo paciente devem ser registradas nos instrumentos utilizados no bloco cirúrgico. Essas anotações possibilitam melhorar a qualidade nas práticas assistenciais, mostrando que há uma correlação positiva entre os registros e a qualidade dos cuidados fornecidos ao paciente (BELO, 2000).

0 registro no relatório de anestesia é uma forma de comunicação escrita entre os profissionais da saúde envolvidos no cuidado ao paciente, pois é no relatório de anestesia que estão contidas as informações sobre o paciente que iram servir para acompanhar a evolução do mesmo (SETZI; D’INNOCENZO, 2009).

Nesse processo, o relatório de anestesia é um instrumento comum, que acompanha o paciente desde sua entrada no bloco cirúrgico, sendo que em alguns casos tem informações obtidas previamente, em consulta prévia do paciente com o anestesiologista. Esse profissional deve preencher o relatório de anestesia, com os dados pertinentes ao paciente e com a devida checagem de medicamentos, considerando que estes possuem alta complexidade. Esses registros, devidamente preenchidos, garantem a qualidade da assistência ao paciente e a segurança do processo conforme as metas de segurança do paciente hospitalizado (MOTTA, 2010).

Popov e Penishe (2009) destacam a importância da qualidade na comunicação, tanto verbal quanto escrita, da equipe multiprofissional, composta pelo anestesiologista, enfermeiro, técnico e auxiliar de enfermagem, sendo atribuição do profissional enfermeiro dar assistência segura, racional e individualizada até que o paciente recupere suas funções fisiológicas. 0 trabalho da equipe é um elemento fundamental para assegurar a qualidade do cuidado com o paciente. Por outro lado, estudos tem mostrado que o déficit de comunicação entre o médico e enfermeiro pode ser um elemento significativo associado à mortalidade de paciente (WRIGHT, 2012).

Assim, considerando a complexidade dos procedimentos clínicos cirúrgicos e do diagnóstico, realizados no bloco cirúrgico, com o uso de diversos fármacos, torna-se imprescindível a existência de um registro adequado.

Nesse contexto, esse estudo teve como objetivo avaliar a qualidade dos registros em relatórios de anestesia utilizados no bloco cirúrgico de um hospital terciário de Porto Alegre.

\section{MÉTODOS}

O estudo é descritivo-exploratório, desenvolvido com base em dados oriundos de bloco cirúrgico de um hospital terciário de Porto Alegre, RS, o qual foi aprovado pelo Comitê de Ética em Pesquisa do Centro Universitário Metodista IPA (número 919.387/2014), de acordo com o Conselho Nacional de Saúde, conforme a Resolução 466/2012. 
A população estudada foi composta por pacientes submetidos a procedimentos cirúrgicos, no segundo semestre de 2014, totalizando 437 pacientes de um hospital terciário, o qual tem a finalidade institucional da realização de diagnóstico, exames e tratamentos de alta complexidade Os instrumentos de pesquisas não foram atribuídos diretamente ao paciente, mas indiretamente por meio de documentos de controle interno no Hospital sendo autorizados pelos responsáveis através do termo de autorização institucional. Assim, na coleta de dados utilizou-se relatório de anestesia (RA), os quais acompanham o paciente submetido à cirurgia, desde seu acesso ao bloco cirúrgico até sua transferência para o leito, no pós-operatório. A partir desse documento as cirurgias foram classificadas de acordo com o tempo de duração em: curto (duração menor que 1 h), médio (duração da cirurgia igual ou maior que $1 \mathrm{~h}$ e menor que $3 \mathrm{~h}$ ), longo (duração igual ou maior que $3 \mathrm{~h}$ ); e tempo não informado.

A coleta dos dados foi obtida com base em cinco abordagens: a) informações pessoais do paciente (idade, sexo, cor, peso, altura, glicemia, creatinina, uréia); b) registro de comunicação prévia com paciente, por meio de anamnese ou observações; c) informações cirúrgicas (data, hora, estimativa de risco - ASA, cirurgia proposta, técnica anestésica, tempo da cirurgia); d) informações clínicas e durante o transoperatório (pressão arterial, agentes e monitores de oxigênio utilizados); e) informações do anestesista e cirurgião.

Em relação a estimativa de risco, de acordo com ASA (2014), a Sociedade Americana de Anestesiologistas classifica o paciente em: ASA I - paciente saudável normal; ASA II - paciente com doença sistêmica leve; ASA III - paciente com doença sistêmica grave; ASA IV - paciente com doença sistêmica grave, que é uma ameaça constante à vida; ASA V - paciente moribundo que não é esperado para sobreviver sem a operação; ASA VI paciente com morte cerebral cujos órgãos estão sendo removidos para fins de doadores.

A análise consistiu na quantificação do número de campos preenchidos. A partir disso, foi verificado o número médio de cirurgias de curto, médio e longo tempo de duração, visando identificar se a abrangência de informações nas RA está associada à complexidade da cirurgia ou ao profissional responsável pelo preenchimento. Paralelamente, informações contidas no RA foram organizadas para análise e descrição. Em relação às informações pessoais dos pacientes a idade foi categorizada em seis classes, visando facilitar a descrição. As informações referenciadas em menos de $20 \%$ das fichas foram apresentadas de forma textual, sem associação quantitativa.

O banco de dados foi organizado no programa Excel e analisado por meio de estatística descritiva. As variáveis foram apresentadas em números absolutos, percentuais, média e desvio padrão. A partir destes resultados realizou-se análise descritiva, a qual tem como característica a inferência sobre dados qualitativos, com finalidade de obter novas reflexões, para adequações e qualificação de instrumentos importantes utilizados no sistema de saúde.

\section{RESULTADOS}

O RA analisado apresenta 50 campos de preenchimento, dos quais cerca de $20 \%$, como glicemia, creatinina, exames de coagulação (TP e KTPP), raramente foram preenchidos. Dos 437 documentos avaliados, aproximadamente $15 \%$ não tiveram o campo referente ao tempo de duração da cirurgia explicitado. A maioria das cirurgias realizadas no período do estudo (57\%) foi de média duração, as quais variaram de uma a três horas, $15 \%$ de curta e 13\% de longa duração (Tabela 1).

Dentre as informações disponíveis sobre o sexo do paciente, constatou-se $56 \%$ das cirurgias realizadas em mulheres e $44 \%$ em homens. Entretanto, quando se considerou o total de documentos analisadas, $36 \%$ não apresentou a informação referente ao sexo do paciente.

Tabela 1. Número de cirurgias de acordo com o porte, relacionado ao tempo de duração, e ao sexo do paciente, em um hospital terciário de Porto Alegre, RS.

\begin{tabular}{lccccc}
\hline \multirow{2}{*}{ Sexo } & \multicolumn{5}{c}{ Duração } \\
\cline { 2 - 5 } & Curta & Médio & Grande & RA & Total \\
\hline \multirow{2}{*}{ Feminino } & 26 & 84 & 18 & 27 & 155 \\
Masculino & 18 & 72 & 16 & 18 & 124 \\
SI & 23 & 94 & 21 & 20 & 158 \\
\hline Total & 67 & 250 & 55 & 65 & 437 \\
\hline
\end{tabular}

$\mathrm{RA}_{\text {inc }}$ : Relatório de anestesia incompleto SI: Sem Identificação

A idade do paciente foi detectada em $58 \%$ dos RA, entre os quais quase a totalidade também apresentou o registro do sexo. Os pacientes apresentavam de 18 a 93 anos, porém a maioria (44\%) destes tinha 60 anos ou mais (Figura 1). 


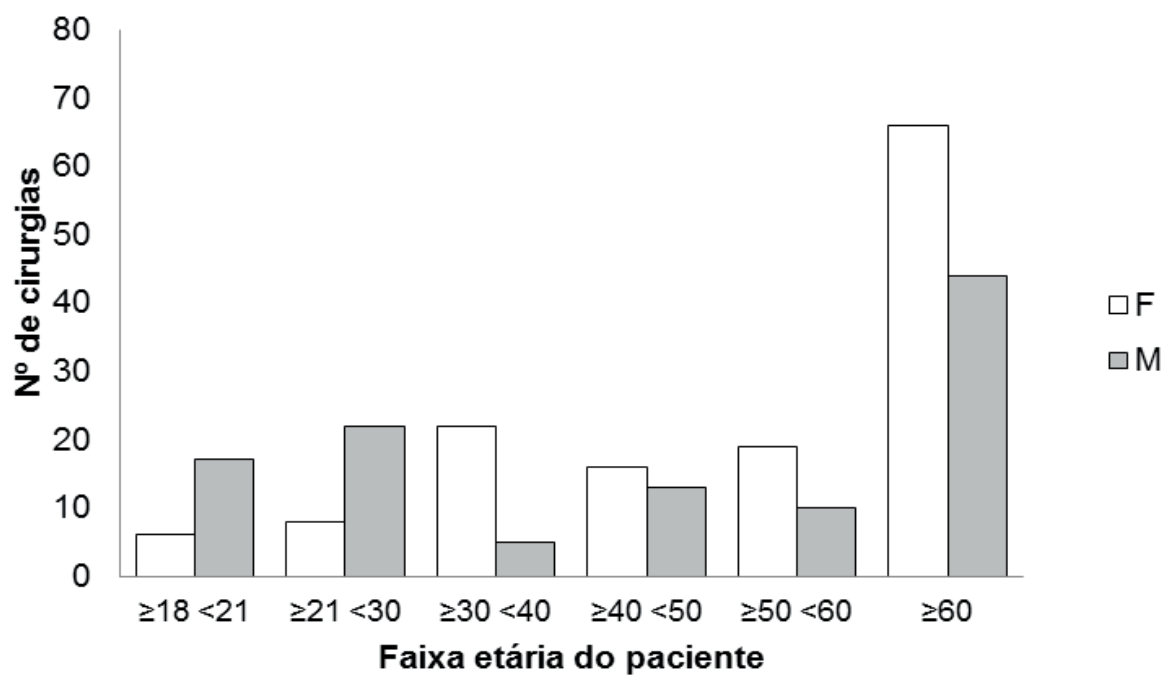

Figura 1. Padrão de faixa etária dos pacientes,relatórios de anestesia foram avaliadas em um hospital terciário de Porto Alegre, RS.

Reduzido número de RA continham informação da cor, peso do paciente e estimativa de risco (ASA), e nenhuma indicou altura, níveis de glicemia, creatinina uréia e outras informações referentes a exames prévios. A anamnese, observada no campo "avaliação pré-operatória" ou "pré-aanestésica" foi realizada e registrada em 269 RA, assim constatando-se que $62 \%$ dos pacientes foram pré-avaliados, por meio de questionamentos antes da cirurgia. Entretanto, a ASA, que utiliza escores para avaliar o risco cirúrgico, foi registrada em menos de $50 \%$ das RA.

Em todos os RA havia registros referentes aos agentes, ou seja, aos fármacos utilizados e dosagens, porém em muitos casos com reduzida legibilidade. A maior atenção também foi dada aos registros de monitoramento de gases, tendo vista que o nível de oxigênio é um dos primeiros procedimentos realizados com o paciente, no leito cirúrgico.

No campo de preenchimento "técnica anestésica indicada" e/ou em outro onde consta "técnica anestésica" a anestesia geral foi a mais utilizadas, seguida por sedação, raqueanestesia, bloqueio plexo e local, sendo mantido incompleto somente em um RA e ilegível em cinco. Destaca-se também que a informação "cirurgia proposta" ou "cirurgia realizada", não foi informada em dois RA e foram ilegíveis em seis documentos.

A pressão arterial foi registrada de forma diferenciada, de acordo com o anestesiologista, sendo que a maio- ria procedeu a análise por meio da mínima (diastólica) e máxima (sistólica), porém também houve registro da média.(pressão arterial média). Esses registros eram feitos a cada cinco minutos, o que permite uma demonstração gráfica e dinâmica ao longo da cirurgia. Foram constatados 372 registros (85\%) com a variação sistólica e diastólica, 53 (12\%) com registro da pressão arterial média e 12 (3\%) sem informações. Algumas situações mostraram oscilação da pressão arterial no decorrer da cirurgia. Com base nos 372 registros que constavam a sistólica e diastólica, no início da cirurgia pode-se considerar que cerca de $10 \%$ dos pacientes apresentavam pressão normal, enquanto, no final da cirurgia $6 \%$.

As maiores médias de pressão arterial foram observadas no início das cirurgias (pressão arterial: sistólica:

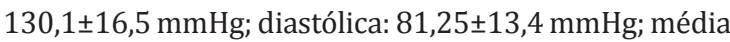
$101,6 \pm 13,4 \mathrm{mmHg}$ ) quando comparado ao término (sistólica: 111,2 $\pm 13,2 \mathrm{mmHg}$; diastólica: $68,7 \pm 13,6 \mathrm{mmHg}$; média $87,6 \pm 12,3 \mathrm{mmHg}$ ), ocasião em que os pacientes são encaminhados à recuperação (pós-operatório).

\section{DISCUSSÃo}

Constatou-se preenchimento inadequado na maioria dos RA, resultado que esteve associado ao profissional responsável, pois foram observados três grupos: aquele que apresentava informações mais completas e legíveis; o que apresentava um nível intermediário de informa- 
ções (maior número de profissionais); e aquele que apresentava poucas informações a respeito das cirurgias. Entretanto, destaca-se que as informações mais presentes eram aquelas referentes ao decorrer da cirurgia, ao contrário das informações prévias sobre a saúde do paciente constatada, por meio de exames (glicemia, creatinina, uréia, entre outras). Percebeu-se que entre os campos não preenchidos estavam aqueles que permitiam suposição, como por exemplo, cor, sexo e duração da cirurgia, considerando que poderiam ser observados visualmente, pelo nome do paciente e pelo gráfico de pressão construído durante a cirurgia, tornando o documento duvidoso. Também foi observada ausência de informação das questões obtidas por questionamento (altura, peso), as quais poderiam inclusive servir para início de um diálogo, conduzindo a um atendimento humanizado.

Mesmo diante da possibilidade das algumas informações estarem presentes em exames realizados previamente, deveriam ser transferidas para o RA, o qual acompanha o paciente e representa um documento do hospital. Conforme Popov e Peniche (2009), a incidência de complicações no pós-operatório está fortemente relacionada com a condição pré-operatória, ou seja, a condição intrínseca ao paciente, e que pode ser conhecida por meio de uma avaliação pré-anestésica adequada.

As cirurgias com tempo médio de duração (uma a três horas) predominaram, com predomínio de pacientes do sexo feminino (Tabela 1), indicando o mais efetivo uso dos serviços por mulheres.

Considerando que a maioria das cirurgias foi realizada em pacientes com mais de 60 anos, e que $40 \%$ dos RA dessa faixa etária não apresentam registros de pré-avaliação, resgata-se um ponto importante a ser refletido, no que se trata do estado psicológico fragilizado do paciente, proporcionado pela ausência de comunicação com um dos principais profissionais responsável pela cirurgia (Figura 1). Associado a isso, estudo de Lenardt et al. (2007) destacou os constrangimentos vividos por idosos durante internações cirúrgicas, em que um dos pontos relatados referia-se ao constrangimento com a equipe de profissionais. Os autores explicaram o fato pelo momento histórico em que nasceram, tendo em vista que vivenciaram repressões e estrutura rígida de valores morais, concluindo sobre a importância do envolvimento e estímulo ao idoso, como protagonista no evento de internação cirúrgica.
Associado a isto Silva e Apolinário (2011) descreveram que o atendimento ao idoso exige uma avaliação ampla e que o atual modelo de atenção oferecido aos pacientes dessa faixa etária apresenta falhas, podendo muitas vezes representar ao paciente hostilidade no atendimento. Tal circunstância poderá ser um agravante à continuidade do atendimento, tendo em vista a ação do emocional sobre a condição fisiológica do paciente.

Porém, não apenas com idosos, pois a pré-avaliação é uma prática importante para qualquer faixa etária e por isso, faz parte do RA. Nesse contexto, salienta-se que a avaliação pré-anestésica, além de proporcionar o máximo possível de informações sobre o paciente, gera um elo de confiança (ORTENZI, 2001). Isso ocorre, porque o paciente sente-se assistido por alguém próximo, com quem conversou e pode contar, durante um momento especial e com risco de vida. Dessa forma, esse procedimento tem uma função técnica e psicológica, ampliando as possibilidades de ações dos profissionais que permanecerão na assistência durante o pós-operatório.

De acordo com ASA (2013) é responsabilidade ética do anestesiologista realizar avaliação pré-operatória, o que facilita o processo de tomada de decisão, especialmente em relação à escolha da técnica anestésica.

Em relação à técnica anestésica de forma semelhante ao presente estudo, Popov e Peniche (2009) verificaram que a anestesia geral foi o procedimento mais frequentes entre as cirurgias. Possivelmente, isso está associado ao porte cirúrgico, que preconiza manter a viabilidade das vias aéreas.

Os valores elevados da sistólica, na fase inicial da cirurgia, possivelmente, esteja associada à maior tensão dos pacientes, nesse momento, pois conforme a Sociedade Brasileira de Cardiologia o valor de pressão arterial, considerado normal é representados por sistólica e diastólica menores de 130 e 85, respectivamente (SBC, 2016). A resistência vascular periférica é uma característica comum em idosos, grupo mais numeroso nos atendimentos cirúrgicos. Silva e Apolinário (2011) relatam que isso ocorre porque com a idade o miocárdio passa a ter perda celular e aumento do tecido conjuntivo, proporcionando enrijecimento valvar e fibrose do sistema de condução e, com isso, a resistência vascular periférica, com consequente hipertensão arterial sistólica, hipotensão ortostática, disfunção distólica e a baixa capacidade de elevação do debito cardíaco devido às situações de estresse. 
De forma geral, foram constatados registros mais efetivos em relação à parte técnica do profissional durante a cirurgia, mas salienta-se que o profissional de saúde deve viabilizar informações precisas e consistentes, de forma favorecer a continuidade da assistência ao paciente, assim, sendo inadequada a omissão de informações nos documentos. $\mathrm{O}$ fato de alguns campos serem presumíveis, não justifica a inobservância ou falta de registro no RA principalmente considerando que esse é o documento que acompanhará o paciente, no pós-operatório.

Imediatamente após a cirurgia, o profissional que recebe o paciente deve estar atento para diversas situações de risco que podem ocorrer. Smeltzer et al. (2011), relataram que a utilização de polifármicos, como por exemplo os opióides, comumente utilizados antes e durante a cirurgia, podem proporcionar intercorrências indesejáveis, sendo que o profissional que atua na assistência deve estar apto a identificar e sanar. Entre os idosos existe uma alta incidência de reações adversas medicamentosas, devido a maior prevalência de polifarmácia (SILVA; APOLINÁRIO, 2011)

Popov e Peniche (2009) destacaram também a necessidade de uma equipe multidisciplinar na fase pós-anestésica, em número suficiente, minimizando problemas maiores proporcionados por complicações, como a dor, hipo e hipertensão, agitação e ansiedade, tremores, náuseas e/ou vômitos, sangramentos, hipoxemia e hipotermia. Entretanto, essas reações podem estar associadas às características do paciente, presentes no RA, mas que por inobservância não foram informadas.

Nesse sentido, considerando-se que os documentos representam registros hospitalares, sua elaboração não foi aleatória, mas construída por meio do conhecimento técnico e situações precedentes que necessitaram tais informações, assim tem-se que os campos devem ser preenchidos. No caso das informações serem excessivas, inadequadas, desnecessárias, deve-se partir da análise conjunta pela equipe de profissionais envolvidos e que utilizam o documento, para sua reelaboração (SEVA-LLOR et al., 2015).

Por outro lado, diante da existência do RA, o qual representa um registro e documento hospitalar, este deve ser completamente preenchido, principalmente quando se considera a complexidade dos procedimentos no centro cirúrgico, sendo foco de constantes e necessárias auditorias (CHICA FERNÁNDEZ et al, 2015).
A qualidade de documentações e registros é fundamental nas ações assistenciais, sendo a forma escrita a mais importante comunicação entre equipes, posteriormente, vindo a auditoria que demanda controle de informações a fim de minimizar erros, indicar falhas e apontar sugestões de melhoria de processos e efetividade de comunicação (FONSECA, 2005; MARTÍNEZ; CANTERO, 2013).

Esses documentos, ainda servem para mediar conflitos, tendo em vista que áreas cirúrgicas são reconhecidas por tal situação, fato ocasionado pela alta exigência de conhecimento, trabalho e responsabilidade na unidade. Esse problema foi pesquisado por Stum; Maçalai; Kirchner (2006), cujo estudo constatou que a condução do relacionamento dos profissionais (cirurgiões, anestesistas, técnicos e auxiliares de enfermagem) representava uma das principais dificuldades enfrentadas por enfermeiros, responsáveis por centros cirúrgicos, atribuindo o fato as diferenças individuais de cada profissional. Assim a documentação escrita, passa ser uma aliada de todas as partes envolvidas (profissionais, pacientes e administração hospitalar).

Um procedimento recente para minimizar problema de comunicação é a informatização (Braga, 2007). De acordo com Crossetti; d'Ávila; Dias (2011) a existência de um prontuário eletrônico tem várias vantagens como: permite acesso rápido aos dados do paciente, registros reais, histórico e legível, o que determina maior segurança ao paciente, reduzindo risco de transcrição de dados.

Contudo, reconhece-se a importância dos documentos, que quando existentes devem ser utilizados e preenchidos em sua íntegra, garantindo assim a qualidade do serviço e da assistência.

\section{CONCLUSÃo}

Constatou-se que a maior proporção dos RA analisados apresenta lacunas de informações, indicando sua baixa qualidade, possível ineficiência e necessidade de reanálise do documento. As informações de âmbito técnico transcorridas durante a cirurgia foram informadas frequentemente, entretanto, aquelas inerentes às avaliações pré-anestésicas foram escassas, indicando reduzida comunicação entre os profissionais de saúde e o paciente. 
Documentos que acompanham o paciente em qualquer unidade do hospital representam uma forma de comunicação dos profissionais, respaldando as ações transcorridas para o setor administrativo e para os pacientes que tem direito de solicitar cópia. Assim, devem ser preenchidos em sua íntegra.

A documentação existente é instrumento de auditorias e avaliação da necessidade de treinamentos, deven- do ser periodicamente revista pelos usuários. A revisão dos documentos deve avaliar pontualmente a pertinência de cada campo de preenchimento, visando evitar questões repetitivas e formulários exaustivos. Isso poderá ser uma estratégia para qualificação de registros hospitalares, tendo em vista que mesmo diante da complexidade do bloco cirúrgico constatou-se inobservância no preenchimento dos documentos analisados. 


\section{REFERÊNCIAS}

ASA. American Society of Anesthesiologists. Asa Physical Status Classification System. 2014. Acesso: 04 de setembro de 2016. Disponível em: https://www.asahq.org/resources/clinical-information/asa-physical-status-classification-system.

ASA. American Society of Anesthesiologists. Guidelines for the ethical practice of anesthesiology. Committee of ethics. 2013.4p.

BELLO, C. N. Recuperação pós-anestésica - escalas de avaliação, princípios gerais. Rev. Centro Est. Anest. FMUSP. v. 9, p. 4-5, 2000.

BRAGA, S. S.Podem as novas tecnologias de informação e comunicação auxiliar na consolidação das democracias? Um estudo sobre a informatização dos órgãos legislativos na América do Sul. Opin. Publica , 2007, 13(1) : 1-50.

CHICA FERNÁNDEZ, M. J.; COSTA RUIZ, A..; RODRÍGUEZ VILLAGORDO, M. J.; SÁNCHEZ SALMERÓN, A. TORRES GÓMEZ, J. F Informe alta enfermería: historia clínica integral. Enfuro 2012; (122):19-24.

CROSSETTI, M. da G.; D’ÁVILA, M.; DIAS, V. L. M. Construção do processo de enfermagem no HCPA e sua informatização. In: ALMEIDA, M. de A. et al. Processo de Enfermagem na Prática Clínica: Estudos clínicos realizados no Hospital de Clínicas de Porto Alegre. Porto Alegre: Artmed. p. 41-51, 2011.

FONSECA A.S., YAMANAKA N.M.A., BARISON T.H.A.S., LUZ S.F. Auditoria e o uso de indicadores assistenciais: uma relação mais que necessária para a gestão assistencial na atividade hospitalar. Mundo Saúde (1995), 29(2):161-169. 2005,

ISSA, M.R.N.; ISONI, N.F.C.; SOARES, A.M.; FERNANDES, M.L. Preanesthesia Evaluation and Reduction of Preoperative Care Costs. Rev Bra Anestesiol, 2011; 61(1): 60-71.

LENARDT M.H., HAMMERSCHMIDT K.S.A., PÍVARO A.B.R., BORGHI A.C.S. Os idosos e os constrangimentos nos eventos da internação cirúrgica. Florianópolis, Texto Contexto Enferm, 2007 Out-Dez; 16(4): 737-45.

MARTÍNEZ, L.; CANTERO, M. L. Informe de cuidados en enfermería: revisión de su situación actual. Enferm Glob. 2013; 12(32):336-45.
MOTTA, A.L.C Auditoria de Enfermagem nos Hospitais e Operadora de Planos de Saúde. 5ª ed. São Paulo: Editora Érica, 2010.220p.

ORTENZI, A, V. Avaliação e medicação pré-anestésica. In: YAMASHITA, A. M.; TAKAOKA. F.; AULER JR., J. O. C.; IWATA. N. M. Anestesiologia. São Paulo: Ed. Atheneu. p. 467-497. 2001.

POPOV, D.C.S.; PENICHE, A. de C.G. As intervenções dos enfermeiros e as complicações em sala de recuperação pós-anestésicas. Rev. Esc. Enferm USP, 2009, 43(4): 953-961.

SBC. Sociedade Brasileira de Cardiologia. Consensos e Diretrizes. SBC \Departamento de Pressão Arterial. Acesso em 01 de agosto de 2016. Disponível em: http://departamentos.cardiol.br/dha/consenso3/capitulo1.asp

SEVA-LLOR, A.M.; MONTESINOS, M.J.L.; ORTEGA, C.B.; CECAGNO, D.; ROCHE, F.P. Relatório de enfermagem no hospital. Acta Paul. Enferm. 2015, 28(2) : 101-6.

SETZI, V.G.; D’INNOCENZO, M. Avaliação da qualidade dos registros de enfermagem no prontuário por meio da auditoria. Acta Paul. Enferm, 2009, 22(3): 313-17.

SILVA, E.M.; APOLINÁRIO, D. Abordagem do idoso no Pronto Socorro. In: MARTINS, H.S. et al. Emergências clínicas: abordagem prática. Barueri, SP: Manole. 2011. p. 281-290.

SMELTZER, S. C.; BARE, B. G.; HINKLE, J. L.; CHEEVER, K. H. BRUNNER E Tratado de Enfermagem Médico-Cirúrgica, 12 ed. Rio de Janeiro, SP: Guanabara Koogan, 2011. 2404p.

SOBECC. Sociedade Braileira de Enfermeiros de Centro Cirúrgico e Recuperação Anestésica e Centro de Material de Esterilização (Sobecc). Práticas recomendadas. 6 ed. São Paulo: SOBECC. 2013. 282p.

SOUZA, R.M. de; CARVALHO, R. de; PALDINO, C.M. Diagnóstico, prognósticos e intervenções de enfermagem na sala de recuperação pós-anestésica. Rev. SOBECC, 2012.17(4): 33-47.

STUMM, E.M.F., MAÇALAI R.T., KIRCHNER R.M. Dificuldades enfrentadas por enfermeiros em um centro cirúrgico. Florianópolis, Texto Contexto Enferm., 2006. 15(3): 464-71.

WRIGTH, D. Trabalho em equipe. In: GEROLIN, F. S. F. Cuidado Baeado no Relacionamento: Um modelo para transformação da prática. São Paulo: Ed. Atheneu. 2012. P.45-61 